\title{
Anterior Cruciate Ligament (ACL) Reconstruction Augmented With Bone Marrow Concentrate, Demineralized Bone Matrix, Autograft Bone, and a Suture Tape (The Fertilized ACL)
}

\author{
Chad Lavender, M.D., Vishavpreet Singh, M.D. (PGY 4), Galen Berdis, M.D. (PGY 4), \\ William Fravel, M.D. (PGY 3), Collin Lamba, M.D. (PGY 2), and Tyag Patel, M.D. (PGY 3)
}

Purpose: To examine the early clinical outcomes as well as safety of the Fertilized anterior cruciate ligament (ACL) reconstruction procedure. Methods: A total of 16 consecutive patients with a mean age of 24 years (range, 16-45 years), who had been treated with the fertilized ACL were evaluated and followed over 2 years. Four patients underwent reconstruction using an FGL GRAFTLINK allograft (LifeNet Virginia Beach, VA) and 12 using quadriceps autografts. All patients received the fertilized ACL as previously described using bone marrow composite, demineralized bone matrix, and an internal brace (Arthrex, Largo, FL). Clinical outcomes at 2 years including International Knee Documentation Committee and Marx scores were evaluated. Complication rates, including return to operating room, arthrofibrosis, infection, and rerupture rates, were also assessed. Results: All patients were followed for 6 months and all were released to full activity. In total, 11 of 16 patients were available for 2 -year follow-up after the fertilized ACL reconstruction. At 2 years, the average International Knee Documentation Committee and Marx scores were 81 (standard deviation, 5.9) and 9 (standard deviation, 5.7), respectively. One patient required a return trip to the operating room for manipulation under anesthesia. No reruptures were observed at the 2-year mark. Nine of 11 patients had returned to their preinjury activity status at 2 years. Conclusions: The fertilized ACL, which adds biology and an internal brace to an ACL reconstruction, is a reliable and safe option when performing an ACL reconstruction. Very low complication risks were seen in this consecutive series followed for 2 years. Patients consistently returned to their preinjury activity status. Level of Evidence: Level IV, therapeutic case series.

$\mathbf{R}$ ecent literature suggests that graft rerupture rates following anterior cruciate ligament (ACL) reconstruction are as high as $6 \%$ to $11 \% .{ }^{1}$ Furthermore, athletes younger than the age of 25 years have been found to have rates of additional ACL injury of up to $23 \%$ when considering both the operative and contralateral sides. ${ }^{2}$ Despite newer techniques and evolutions in graft

From Marshall University, Scott Depot, West Virginia, U.S.A.

The authors report the following potential conflicts of interest or sources of funding: C.L. is a consultant for arthrex outside of this submitted work. Full ICMJE author disclosure forms are available for this article online, as supplementary material.

Received March 8, 2021; accepted July 30, 2021.

Address correspondence to Chad Lavender, M.D., Marshall University, 300 Corporate Center Dr., Scott Depot,WV 25560.E-mail:doclav@gmail.com

(C) 2021 THE AUTHORS. Published by Elsevier Inc. on behalf of the Arthroscopy Association of North America. This is an open access article under the CC BY-NC-ND license (http://creativecommons.org/licenses/by-nc-nd/4.0/). 2666-061X/21311

https://doi.org/10.1016/j.asmr.2021.07.030 choices, significant reductions in rerupture rates and return-to-play time have been elusive. Bone marrow concentrate (BMC) has shown promise in the treatment of many orthopaedic conditions, including osteochondral injuries, avascular necrosis, and tendon injuries. ${ }^{3}$ The literature on BMC and demineralized bone matrix in ACL reconstruction is sparse, but BMC has been found to improve biologic incorporation in osteochondral allografts. ${ }^{4}$ To encourage early biologic graft incorporation and prevent early graft rerupture, a technique was recently created for augmentation of bone-patella tendon-bone ACL reconstruction with BMC and suture tape. ${ }^{5}$ This technique also can be used to enhance an all-inside ACL reconstruction, which allows grafting of both the femoral and tibial tunnels with BMC and demineralized bone matrix. Furthermore, a technique was recently described recovering the reamings of the femoral and tibial tunnels for use as autograft using the GraftNet Autologous Tissue Collector (Arthrex, Naples, FL). ${ }^{6}$ 
Table 1. Clinical Results

\begin{tabular}{|c|c|c|c|c|c|}
\hline Graft Type 2 & Age, $y$ & Preinjury Activity? (Y or N) & Retear? (Y or $\mathrm{N}$ ) & IKDC Score & Marx Score \\
\hline Allograft & 37 & $\mathrm{~N}$ & $\mathrm{~N}$ & 84 & 8 \\
\hline Allograft & 38 & & & & \\
\hline Quad autograft & 18 & $\mathrm{~N}$ & $\mathrm{~N}$ & 83 & 1 \\
\hline Quad autograft & 20 & & & & \\
\hline Quad autograft & 19 & & & & \\
\hline Quad autograft & 21 & $\mathrm{Y}$ & $\mathrm{N}$ & 85 & 15 \\
\hline Quad autograft & 19 & $\mathrm{Y}$ & $\mathrm{N}$ & 81 & 12 \\
\hline Quad autograft & 20 & & & & \\
\hline Quad autograft & 18 & $\mathrm{Y}$ & $\mathrm{N}$ & 74 & 6 \\
\hline Quad autograft & 18 & & & & \\
\hline Quad autograft & 30 & $\mathrm{Y}$ & $\mathrm{N}$ & 67 & 12 \\
\hline Quad autograft & 18 & $\mathrm{Y}$ & $\mathrm{N}$ & 87 & 16 \\
\hline Quad autograft & 20 & $\mathrm{Y}$ & $\mathrm{N}$ & 77 & 12 \\
\hline Allograft & 32 & $\mathrm{Y}$ & $\mathrm{N}$ & 85 & 16 \\
\hline Allograft & 47 & $\mathrm{Y}$ & $\mathrm{N}$ & 84 & 0 \\
\hline Quad autograft & 21 & $\mathrm{Y}$ & $\mathrm{N}$ & 83 & 5 \\
\hline
\end{tabular}

IKDC, International Knee Documentation Committee; N, no; Y, yes.

The possible advantages of these augmentation procedures for ACL reconstruction are numerous but could include improved bony consolidation decreased pain, increased stability, and lower rerupture rates. In this study, we retrospectively reviewed the early clinical outcomes up to 2 years in patients who underwent the fertilized ACL reconstruction, which refers to adding AlloSync Pure (demineralized bone matrix; Arthrex), autograft bone, and BMC to the tunnels of an all-inside ACL reconstruction. The technique also adds an InternalBrace (Arthrex) to the construct. This study is important to perform to evaluate the safety and results of a procedure without long-term prospective data at this point. The purpose of this study is to examine the early clinical outcomes as well as safety of the fertilized ACL reconstruction procedure. We hypothesized that the fertilized ACL reconstruction would provide improved outcomes such as rerupture rates, healing, and activity while having an appropriate safety profile to that of current and historical ACL reconstruction techniques.

\section{Methods}

We obtained institutional review board approval to retrospectively review 16 consecutive patients who underwent the fertilized ACL reconstruction performed between July 2018 and February 2019. Inclusion criteria for the study were patients with a defined ACL tear based on clinical examination and magnetic resonance imaging testing who received a fertilized ACL reconstruction between the defined dates previously. Exclusion criteria included revision ACL reconstructions, standard nonfertilized ACL reconstructions, and patients outside of the consecutive time period. Patients suffering an ACL injury requiring surgery were identified. All patients had a positive Lachman examination preoperatively and were able to perform sporting activities. All patients received the fertilized ACL technique with AlloSync Pure (Arthrex), BMC augmentation, and an internal brace (Arthrex) as previously described. ${ }^{5}$ Then, 60 cc of bone marrow was aspirated from the proximal lateral tibia before inflation of the tourniquet. This is concentrated to 3 cC of BMC. Either a quad tendon autograft or FGL GRAFTLINK allograft (LifeNet Virginia Beach, VA) construct was made on the back table with appropriate all inside fixation devices applied. On the autografts, a FiberTag (Arthrex) was applied to each end and attached to an RT TightRope device (Arthrex) on the femoral side and ABS (Arthrex) on the tibial side of the graft. On the allografts, a BTB TightRope (Arthrex) was placed onto the femoral end of the graft and an ABS (Arthrex) was placed onto the tibial side. The femoral TightRopes were lengthened to allow injection of the composite graft into the femoral tunnel through the medial portal later in the case. Standard notchplasty and preparation was performed for the ACL reconstruction. All-inside tunnels were drilled with the FlipCutter (Arthrex). During drilling of the femoral tunnel, a shaver was used with a GraftNet (Arthrex) to harvest autograft bone. Then, 3 cc of the BMC was added to 5 cc of AlloSync Pure (Arthrex) and 1 cc of the autograft bone to create the composite graft. After passing the femoral button, the composite graft was injected into the femoral tunnel and then down through the tibial tunnel and a standard all-inside ACL was performed as the graft was brought up into the femoral tunnel and down into the tibia. After a button was applied to the tibia, the internal brace was placed into a SwiveLock (Arthrex) in the medial tibia and tensioned at $0^{\circ}$ of extension. All surgeries were performed by the senior author C.L., who is a sports fellowship-trained orthopaedic surgeon. Patients' clinical outcomes at 2 years were measured by an International Knee Documentation Committee (IKDC) subjective score, with 87 as the greatest rating and Marx 


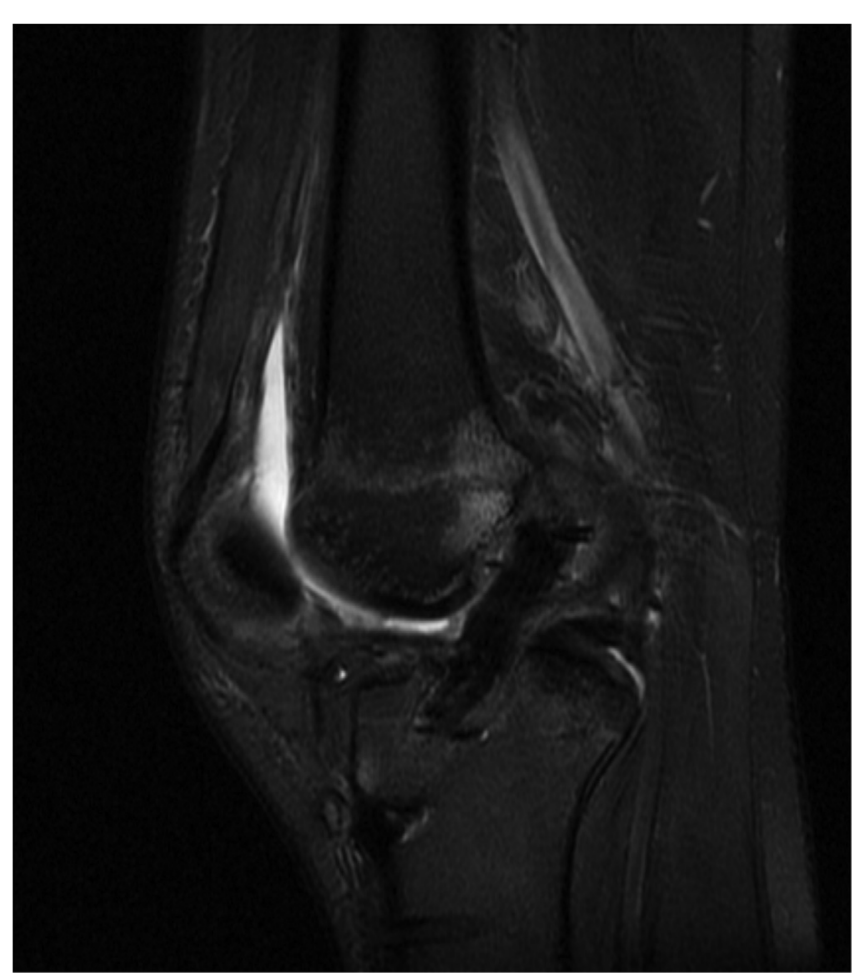

Fig 1. Left knee sagittal magnetic resonance image obtained at 9 weeks showing excellent graft consistency and bony healing of the tibial tunnel which was drilled.

score with 16 as the greatest possible score. Complications including reoperation rates, arthrofibrosis, infection, and rerupture were evaluated. The patients were also asked do they feel their knee was functioning bad, average, or good.

\section{Results}

\section{Clinical Results}

Sixteen patients were followed with radiographs and clinical examinations for 6 months' postprocedure.
Twelve patients underwent quadriceps tendon autograft ACL reconstruction, and 4 patients underwent allograft. Patient's ages ranged between 16 and 45 years, with the average age being 24 years old. Five patients were female and 11 were male. All 16 patients were released to full activity at 6 months. They all had negative Lachman and pivot shift examinations and reported no subjective instability. Eleven of 16 patients were available for follow up at 2 years. The average IKDC score was $81 / 87$ (standard deviation, 5.9) and average Marx score was 9 (standard deviation, 5.7) (Table 1), All patients reported that their knee felt overall average or good, with 7 stating their knee felt good. No patient sustained a rerupture of the graft. One patient had arthrofibrosis and at 6 weeks postoperatively had a manipulation under anesthesia. There were no infections. Nine of 11 patients reported returning to their preoperative activity at 2 years. Radiographs taken at 6 months postoperatively did not show tunnel widening, defined by appearance of a tunnel greater than $2 \mathrm{~mm}$ from what was drilled (Fig 1 ). During the follow-up period, 2 patients had pain and required magnetic resonance imaging. These are included for observation and clearly show consolidation of the tunnels at different time points with excellent graft consistency (Figs 2 and 3).

\section{Discussion}

This series reveals the fertilized ACL is safe and reliable and very comparable with other ACL techniques. We also showed high subjective scores at early 2 -year follow-up and no reruptures. As mentioned, rates of reruptures can vary in the literature and are reported as high as $6 \%$ to $11 \% .^{1}$ All-inside ACL reconstruction has been shown to be equivalent to the full tibial tunnel technique in functional outcome measures with the possible advantage of decreased pain based on visual analog scale. ${ }^{7}$ When used with our technique, the
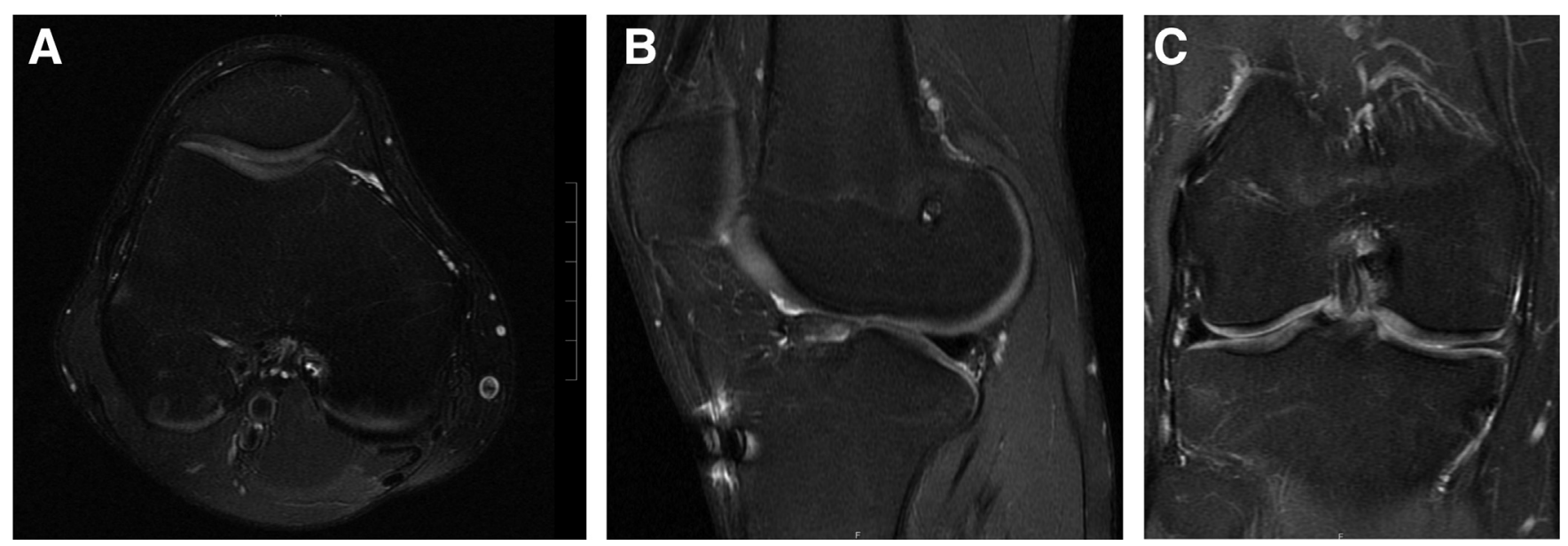

Fig 2. Right knee (A) axial, (B) sagittal, and (C) coronal magnetic resonance images of the femoral tunnel at 2 years showing a nearly complete remodeling of the femur to host bone with no obvious tunnel. 


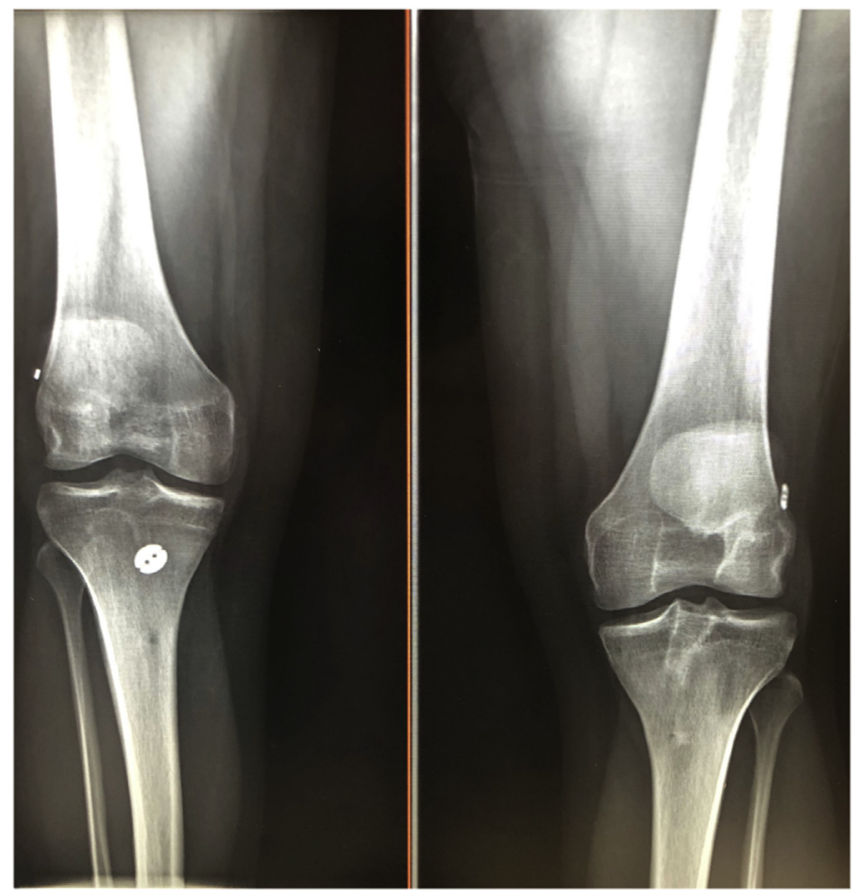

Fig 3. Anteroposterior view of bilateral knees. Patient is 2 years status post left knee BTB anterior cruciate ligament reconstruction with obvious tunnel lysis and widening and is 6 months status post right knee quad tendon fertilized anterior cruciate ligament with barely visible tunnels.

all-inside approach allows for BMC grafting of both the femoral and tibial tunnels, which we hypothesized would aid in early biologic graft incorporation, less tunnel widening, and more substantial graft incorporation on both the femoral and tibial sides. The current study shows a low complication rate with our technique. Patients' self-reported return to previous activity also was very high. Our IKDC of 81 of 87 or $93 \%$ compares favorably with those previously reported in the literature with similar quad tendon ACL reports. Galan et al. $^{8}$ showed $59 \%$ of patient to have normal IKDC scores; however, that study had a larger population and followed patients for 5 years. We feel this is the first step to show the fertilized ACL can be a great addition to the all inside ACL technique in the future. The fertilized ACL procedure is shown in this study to be safe with no increase in early complications in addition to showing no increase in early rerupture rate versus historical data.

There is a $\$ 1,000$ to $\$ 2,000$ additional cost when performing this technique to a standard ACL reconstruction. This is variable across different operating rooms and areas but obviously is considerable. Because of the limitations of our study, further study is warranted. A randomized control trial comparing the fertilized ACL with a standard ACL reconstruction is currently underway to further study possible differences in outcomes. This trial will focus on radiographic findings as well as clinical follow-up.

\section{Limitations}

There are limitations of this study primarily related to the retrospective nonrandomized nature of the study. One of the major limitations of the study is the lack of a control group. Other limitations are the small sample size and early follow-up. We recognize complications such as reruptures and instability could be long term and not found during this early follow up period. This study is also limited by the $31 \%$ dropout rate and by no objective evaluation after 6 months.

\section{Conclusions}

The fertilized ACL, which adds biology and an internal brace to an ACL reconstruction, is a reliable and safe option when performing an ACL reconstruction. Low complication rates were seen in this consecutive series followed for 2 years. Patients consistently returned to their preinjury activity status.

\section{References}

1. Crawford SN, Waterman BR, Lubowitz JH. Long-term failure of anterior cruciate ligament reconstruction. Arthroscopy 2013;39:1566-1571.

2. Wiggins AJ, Grandhi RK, Schneider DK, Stanfield D, Webster KE, Myer GD. Risk of secondary injury in younger athletes after anterior cruciate ligament reconstruction: A systematic review and meta-analysis. Am J Sports Med 2016;44:1861-1876.

3. Imam MA, Mahmoud SSS, Holton J, Abouelmaati D, Elsherbini Y, Snow M. A systematic review of the concept and clinical applications of bone marrow aspirate concentrate in orthopaedics. SICOT J 2017;3:17.

4. Oladeji LO, Stannard JP, Cook CR, et al. Effects of autogenous bone marrow aspirate concentrate on radiographic integration of femoral condylar osteochondral allografts. Am J Sports Med 2017;45:2797-2803.

5. Lavender C, Johnson B, Kopiec A. Augmentation of anterior cruciate ligament reconstruction with bone marrow concentrate and a suture tape. Arthrosc Tech 2018;7: e1289-e1293.

6. Lavender C, Johnson B, Singh V, Dennis E, Torres L. The Lavender fertilized anterior cruciate ligament reconstruction: A quadriceps tendon all-inside reconstruction fertilized with bone marrow concentrate, demineralized bone matrix, and autograft bone. Arthrosc Tech 2019;8: e1019-e1023.

7. Lubowitz JH, Schwartzberg R, Smith P. Randomized controlled trial comparing all-inside anterior cruciate ligament reconstruction technique with anterior cruciate ligament reconstruction with a full tibial tunnel. Arthroscopy 2013;29:1195-1200.

8. Galan H, Escalante M, Della Vedova F, et al. All inside full thickness quadriceps tendon ACL reconstruction: Long-term follow-up results. J Exp Orthop 2020;7:13. 